Ann. Zootech., I973, $22(4), 453-462$.

\title{
EFFETS RESPECTIFS DES NIVEAUX ALIMENTAIRES PENDANT LA CROISSANCE ET LE PREMIER MOIS DE GESTATION SUR LES PERFORMANCES DE REPRODUCTION \\ GHEZ LA TRUIE NULLIPARE : RÉSULTATS PRÉLIMINAIRES
}

\author{
M. ÉTIENNE et P.-H. DUÉE \\ avec la collaboration technique de G. ConseIL \\ Station de Recherches sur l'Élevage des Porcs, \\ Centre national de Recherches zootechniques, I. N.R. A., \\ 78350 Jouy en Josas
}

\section{RÉSUMÉ}

L'expérience porte sur 2 lots de $\mathrm{I} 2$ truies Large White soumises, à partir d'un poids vif de $55 \mathrm{~kg}$, soit à un niveau alimentaire libéral (consommation maximale : 3,28 $\mathrm{kg}$ par jour), soit à une restriction d'environ $45 \mathrm{p}$. Ioo de la quantité d'aliment consommé. A la puberté, les truies sont saillies et chaque lot est divisé en 2 groupes de 6 animaux, l'un conservant le même niveau alimentaire, l'autre soumis au niveau inverse pendant le premier mois de gestation.

Aucune interaction significative n'est notée entre les effets des niveaux de consommation avant et après la saillie.

Une sous-alimentation pendant la croissance retarde de 2 mois l'âge à la puberté des truies ( 363 contre 296 jours) et abaisse leur poids à ce stade. De plus, le taux d'ovulation des truies restreintes est diminué par rapport aux autres animaux ( 12,0 contre 13,8 ), ce qui se répercute sur le nombre d'embryons viables à 3 o jours de gestation $(9,0$ contre 12,5$)$. La mortalité embryonnaire apparaît plus élevée dans le lot de truies restreintes avant la puberté (26,o contre 8,8 p. Ioo).

Le niveau alimentaire de gestation ( $\mathrm{I}, 8$ ou $3,5 \mathrm{~kg}$ par jour) n'a pas d'effet sur le nombre, ni sur le poids des embryons 30 jours après l'accouplement. Par contre, au même stade, le poids moyen des corps jaunes diminue de $132 \mathrm{mg}$ avec le niveau de consommation, de même que la quantité d'annexes disponibles par embryon (membranes placentaires, liquides allantoïdiens).

\section{INTRODUC'TION}

L'influence de l'alimentation pendant la période de croissance sur la maturité sexuelle et sur les performances ultérieures de reproduction n'a pas fait l'objet d'études très détaillées chez la Truie. Aussi, l'alimentation des jeunes reproducteurs 
n'est-elle pas, en pratique, différente de celle des animaux de même âge élevés à des fins de production de viande.

Plusieurs auteurs ont, toutefois, montré que des truies ayant reçu à volonté un régime riche en énergie pendant leur croissance sont plus lourdes à la puberté que des truies soumises à une sous-alimentation (GOSSETT et SøRENSEN, I959; HAINEs et al., I959 ; LODGE et McPherson, I96I). En ce qui concerne l'âge à la puberté, les opinions sont contradictoires. Ainsi, selon SøRENSEN et al. (I96I), une diminution de 1'ingéré énergétique de $5^{\circ} \mathrm{p}$. Ioo n'affecte pas 1'âge à la puberté, ce qui se traduit, évidemment, par une diminution de poids au premier œstrus pour le groupe restreint. Par contre, selon HaInes et al. (I959), la diminution de l'apport d'énergie à partir de $50 \mathrm{~kg}$ de poids vif provoquerait un retard de puberté. A l'opposé, les truies restreintes seraient plus jeunes à la puberté selon les expériences de SELF et al. (I955).

La présente expérience a pour but d'étudier les effets d'une carence alimentaire à partir d'un poids vif de $55 \mathrm{~kg}$, sur l'apparition de la puberté et le taux d'ovulation au premier œstrus. De plus, les animaux ayant été saillis au premier œestrus, les conséquences sur les performances de reproduction à 30 jours de gestation seront recherchées. Enfin, les effets sur les performances de reproduction seront également considérés dans le cas d'une modification du niveau alimentaire après la saillie.

\section{TABI EAU I}

Composition et analyse du régime

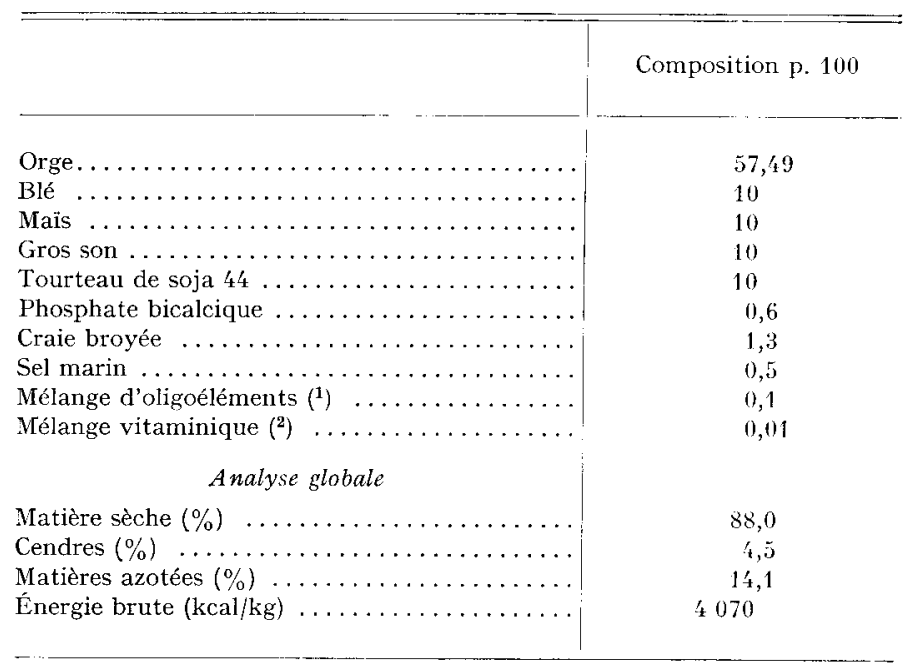

(1) En g pour $1 \mathrm{~kg}$ de mélange : sulfate de fer : 400 ; sulfate de manganèse : 160 ; Sulfate de cuivre : 40 ; Carbonate de zinc : 125 ; Sulfate de cobalt : 0,5 ; Iodure de potassium : 0,3 ; Craie broyée, Q. S. P.

(2) Vitamines par kg d'aliment : vitamine A : 4000 U. I. ; Vitamine $D_{3}$ : 1000 U. I. ; Riboflavine : $2 \mathrm{mg}$; Pantothénate de $\mathrm{Ca}: 5 \mathrm{mg}$; acide nicotinique : $10 \mathrm{mg}$; Choline : $100 \mathrm{mg}$; vitamine $\mathrm{B}_{12}: 0,01 \mathrm{mg}$. 


\section{MODALITÉS EXPÉRIMENTALES}

- Dans une porcherie dite "d'engraissement "sur béton, comportant des loges collectives de 6 animaux, deux lots de 22 porcs femelles Large White, d'un poids initial de 55 kg à l'âge moyen de I55 jours, sont soumis, jusqu'à la puberté, à une échelle de rationnement qui correspond soit à un niveau alimentaire libéral (lot $\mathrm{H}$ ), soit à une restriction alimentaire globale, d'environ $45 \mathrm{p}$. Ioo du niveau précédent (lot B). Un régime unique, dont la composition et l'analyse globale sont rapportées dans le tableau $\mathrm{I}$, est utilisé durant toute l'expérience.

La maturité sexuelle est détectée à partir du moment où le poids moyen des animaux de chaque loge atteint $75 \mathrm{~kg}$ par leur présentation quotidienne au verrat. A la puberté, les truies sont saillies par un même mâle Large White et, pendant 30 jours de gestation environ, les animaux reçoivent le régime précédent au même niveau alimentaire que pendant la période prépubérale (lots $\mathrm{BB}$ et $\mathrm{HH}$ ), ou à un niveau alimentaire différent (lots $\mathrm{BH}$ et $\mathrm{HB}$ ). Le schéma expérimental ainsi que les consommations moyennes des animaux aux différents stades de l'étude sont consignés dans le tableau 2.

TABLEAU 2

Schéma expérimental et consommation moyenne des animaux

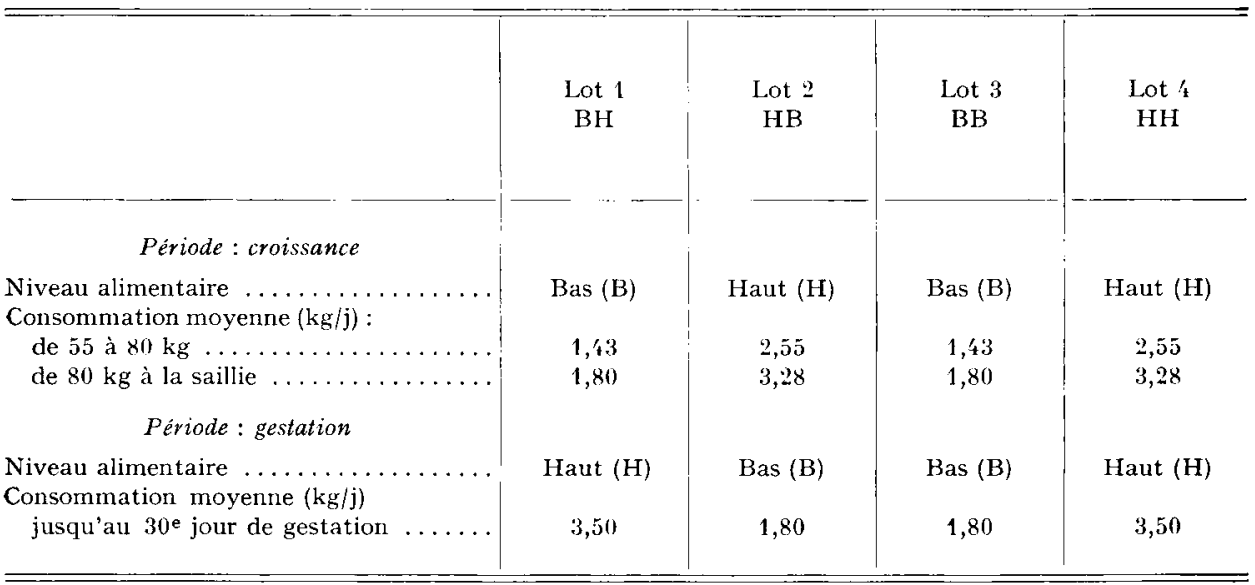

- Toutes les truies sont abattues entre 27 et 33 jours de gestation, et les mesures suivantes sont effectuées : poids des ovaires, nombre et poids des corps jaunes disséqués, nombre et poids moyen des embryons viables, poids des membranes placentaires et des liquides allantoïdiens.

L'étude statistique des différents critères est réalisée en tenant compte des effets des traitements avant et après saillie et de leur interaction éventuelle. D'autre part, le poids moyen des embryons et celui des membranes placentaires sont ajustés à un stade fixe d'abattage : 30 jours de gostation, après correction suivant des coefficients établis par LEGAULT et LEUILLET (I973) et qui font intervenir en plus du stade réel de gestation, le nombre d'embryons de chaque corne utérine.

\section{RÉSULTATS}

\section{I. - Age et poids à la puberte}

Le rationnement différent entre les deux groupes de $\mathrm{I} 2$ truies, à partir d'un poids moyen de $55 \mathrm{~kg}$, permet de distinguer très nettement les animaux sur le plan de la croissance. Ainsi, le gain moyen quotidien jusqu'à la puberté est deux fois plus élevé 
pour le groupe de truies dont l'alimentation est libérale (tabl. 3). Le ralentissement de la croissance observé dans l'autre groupe d'animaux a pour effet de retarder l'apparition de la maturité sexuelle d'environ deux mois. De plus, à ce stade, les truies restreintes sont significativement moins lourdes que les truies de 1'autre lot. Enfin, si

TABLEAU 3

Influence du niveau alimentaire, à partir de $55 \mathrm{~kg}$ de poids vif, sur l'apparition de la puberté et le taux d'ovulation chez la Truie

Age initial (à $55 \mathrm{~kg}$ ) : I 55 jours

Nombre d'animaux par lot : I 2

\begin{tabular}{|c|c|c|c|}
\hline Niveau alimentaire & Haut & Bas & $\begin{array}{l}\text { Signification } \\
\text { statistique } \\
\left.\mathrm{S} \bar{x}(\quad)^{1}\right)\end{array}$ \\
\hline $\begin{array}{l}\text { Gain moyen quotidien }(\mathrm{g}) \text { de } 55 \mathrm{~kg} \\
\text { à la puberté } \ldots \ldots \ldots \ldots \ldots \ldots \ldots \ldots\end{array}$ & fos? & 301 & \\
\hline $\begin{array}{l}\text { Nombre de truies pubères avant } \\
\quad 400 \text { jours d'âge } \ldots \ldots \ldots \ldots \ldots \ldots\end{array}$ & 12 & 10 & \\
\hline Age moyen à la puberté (jours) & 296 & 363 & $6(6,7)^{* * *}$ \\
\hline Poids moyen à la puberté $(\mathrm{kg}) . .$. & 147,1 & 120,6 & $3,6(9,3)^{* * *}$ \\
\hline \multicolumn{4}{|l|}{ Taux d'ovulation $\left({ }^{2}\right)$} \\
\hline $\begin{array}{l}\text { ovaire gauche } \ldots \ldots \ldots \ldots \ldots \ldots \\
\text { ovaire droit } \ldots \ldots \ldots \ldots \ldots \ldots\end{array}$ & $\begin{array}{l}6,7 \\
7,1\end{array}$ & $\begin{array}{l}7,5 \\
4,5\end{array}$ & \\
\hline total $\ldots$ & 13,8 & 12,0 & $0,6(16,0)^{*}$ \\
\hline
\end{tabular}

(1) Écart-type de la moyenne (coefficient de variation).

* Différence significative au seuil $P \leqslant 0,05$;

*** Différence significative au seuil $P \leqslant 0,001$.

(2) Taux d'ovulation estimé par le nombre de corps jaunes, compté à 30 jours de gestation.

tous les animaux du premier lot (lot $\mathrm{H}$ ) atteignent effectivement le stade pubère, il n'en est pas de même dans le lot $B$ où 2 truies, sur les $I 2$ initialement mises en expérience, doivent être éliminées, n'étant pas pubères avant 400 jours d'âge.

Finalement, le premier effet d'une restriction alimentaire pendant la croissance est d'accroître la période improductive des truies.

\section{2. - Taux d'ovulation}

L'estimation du taux d'ovulation par le nombre de corps jaunes comptés au moment de l'abattage à 30 jours de gestation est rapportée dans le tableau 3 .

Une alimentation libérale pendant la croissance accroît significativement de I,8 le nombre d'ovules pondus au premier ostrus, c'est-à-dire le nombre potentiel d'embryons, après fécondation de ces ovules. Il est à noter que cette différence porte, uniquement, sur le taux d'ovulation de l'ovaire droit.

Il a été possible de distinguer dans cette augmentation du taux d'ovulation, l'effet 
propre du poids de la Truie et l'effet dî au traitement. En effet, une relation linéaire significative existe entre le taux d'ovulation $(\mathrm{V})$ et le poids de la femelle au moment du premier ostrus $(\mathrm{X}$, en $\mathrm{kg})$ :

$$
\mathrm{Y}=0,059 \mathrm{X}+4,99(r=0,53) \text {. }
$$

En poursuivant l'analyse statistique par l'ajustement du taux d'ovulation à un poids vif constant ( $135 \mathrm{~kg}$ dans le cas présent), les différences entre les valeurs moyennes ajustées ne sont plus significatives ( 12,7 dans le lot restreint, I3, I dans le lot libéral).

Par contre, aucune relation de ce type n'a pu être établie entre le taux d'ovulation et 1'âge.

En conséquence, l'accroissement du nombre d'ovules pondus sous l'influence du niveau alimentaire semble obtenu par le biais d'une augmentation du poids de l'animal au moment de l'cestrus.

\section{TABLEAU 4}

Influence du niveau alimentaire, avant et après saillie, sur les performances de reproduction estimées à 30 jours de gestation chez la Truie

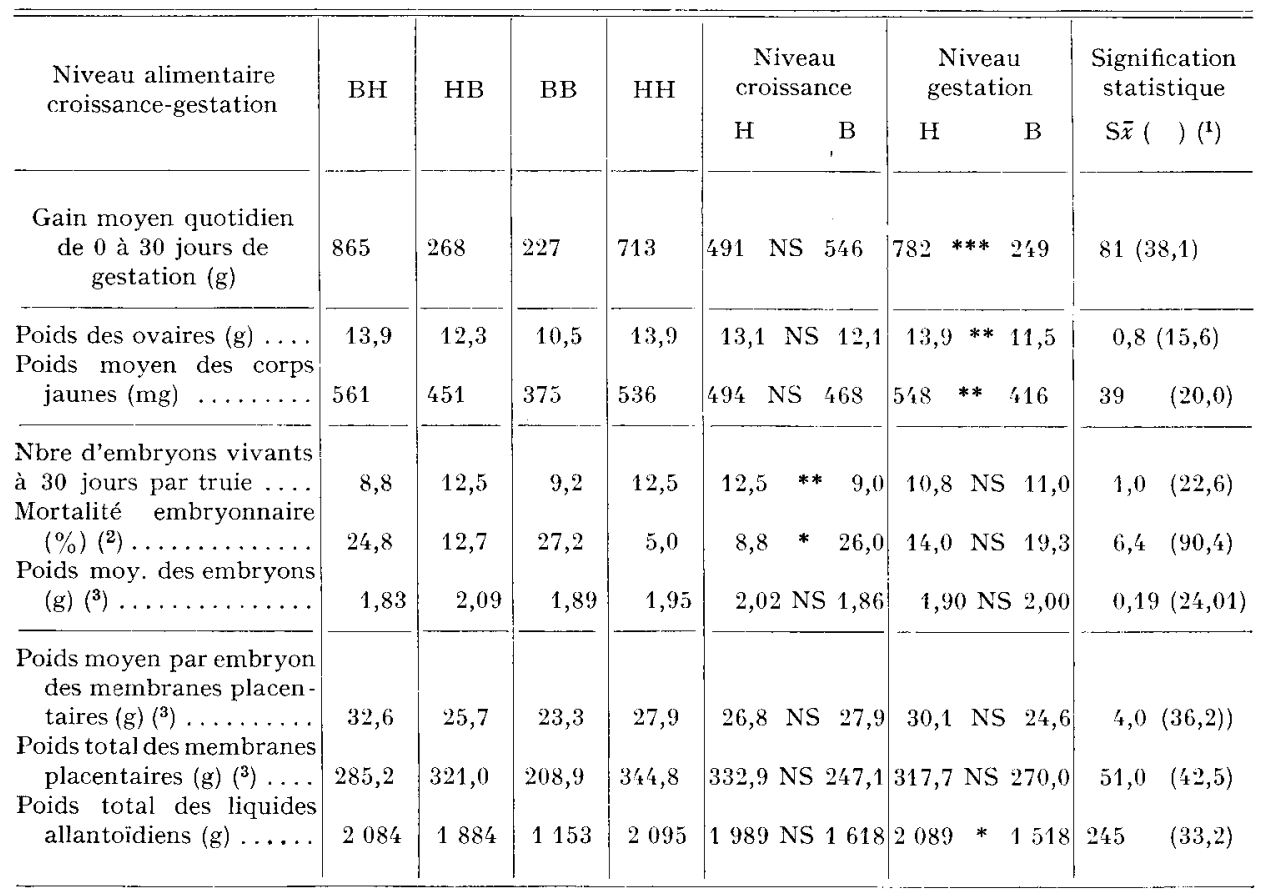

(1) Écart-type de la moyenne (coefficient de variation).

NS : différence non significative au seuil $\mathrm{P} \leqslant 0,05,^{*}$ : différence significative au seuil $\mathrm{P} \leqslant 0,05$.

** : différence significative au seuil $\mathrm{P} \leqslant 0,01,{ }^{* * *}:$ différence significative au seuil $\mathrm{P} \leqslant 0,001$.

Pour tous les critères, interaction niveau croissance $\times$ niveau gestation non significative.

(2) D'après le nombre de corps jaunes équivalent, par hypothèse, au nombre d'ovules fécondés.

(3) Après correction à 30 jours de gestation, d'après des coefficients établis par LEGAULT et LeUillet (1973). 


\section{3. - Gain de poids des truies pendant le premier mois de gestation}

Toutes les truies pubères ont été saillies avec succès et réparties suivant 4 lots définis dans le schéma expérimental précédent (tabl. 2). Une réduction du niveau alimentaire de gestation (de 3,5 à $\mathrm{I}, 8 \mathrm{~kg}$ par jour) diminue très significativement le gain moyen quotidien des animaux, qui passe, en moyenne, de 782 à $249 \mathrm{~g}$ par jour, quel que soit le niveau de consommation avant la saillie (tab1. 4).

\section{4. - Poids des ovaires et poids moyen des corps jaunes à l'abattage}

Les différences enregistrées dans le poids des ovaires (tabl. 4) sont dues, uniquement, au niveau alimentaire de gestation ; en effet, une restriction alimentaire pendant cette période fait baisser le poids des tissus ovariens totaux de $2,4 \mathrm{~g}\left(\mathrm{I}_{3}, 9\right.$ à II,5). Cette diminution peut s'expliquer, en partie, par une diminution parallèle du poids moyen des corps jaunes de $132 \mathrm{mg}$ ( 548 à $4 \mathrm{I} 6 \mathrm{mg}$ ). Si, dans les deux cas, aucun effet spécifique du régime avant la puberté n'a été mis en évidence, on peut noter, toutefois, que les effets néfastes du régime de gestation sur les poids précédents, sont plus nuancés quand le niveau alimentaire avant la saillie est libéral.

\section{5. - Nombre et poids moyen des embryons viables à 30 jours de gestation}

Les résultats rapportés dans le tableau 4 indiquent que le niveau alimentaire avant l'œstrus a un effet direct sur le nombre d'embryons à 30 jours de gestation. L'accroissement de 3,5 embryons viables avec un niveau élevé d'alimentation avant la saillie s'explique partiellement par l'augmentation déjà signalée du taux d'ovulation De plus, une carence alimentaire avant la saillie favorise la mortalité embryonnaire pendant la gestation qui suit (26,o contre 8,8 p. I0o).

Par contre, le niveau alimentaire avant ou après la saillie n'a pas d'effet sur lt poids moyen des embryons corrigé à 30 jours de gestation.

\section{6. - Poids des annexes embryonnaires}

Les valeurs observées pour le poids moyen par embryon des membranes placentaires, corrigé à 30 jours de gestation, ne font pas apparaître d'effet spécifique d'un des traitements avant ou après la saillie. Cependant, ce poids a tendance à augmenter avec un accroissement du niveau alimentaire de gestation $(24,6$ à $30, \mathrm{I} \mathrm{g})$. Parallèlement, une diminution significative de la quantité totale de liquides allantoïdiens est enregistrée avec le niveau bas en gestation ( $15 \mathrm{I} 8$ contre $2089 \mathrm{~g}$ ).

\section{DISCUSSION E'T CONCLUSIONS}

L'analyse statistique des résultats à l'abattage des animaux (30 jours de gestation) ne fait ressortir aucune interaction significative entre les régimes alimentaires avant et après la saillie, ce qui permet, dans une certaine mesure, de dissocier les effets respectifs sur les performances de reproduction des niveaux au cours des deux périodes. 


\section{I. - Influence du niveau alimentaire pendant la période prépubérale}

Il apparaît, tout d'abord, que l'âge moyen à la puberté des truies Large White est plus élevé dans cette expérience que celui enregistré par L EGAULT (I973) sur un grand nombre d'animaux de même race ou par d'autres auteurs sur des reproducteurs de races différentes (SElF et al., I955; HaInes et al., I959). Ce retard au moment de la maturité sexuelle peut s'expliquer, en partie, par un retard initial de croissance à la mise en lots (âge élevé correspondant au poids de départ de $55 \mathrm{~kg}$ ). Mais l'état sanitaire des animaux utilisés était satisfaisant, ainsi qu'en témoigne leur vitesse de croissance élevée atteinte pendant la gestation, lorsqu'ils reçoivent $3,5 \mathrm{~kg}$ d'aliment par jour. D'ailleurs, les femelles du lot BH réalisent une croissance compensatrice après la saillie par rapport à celles du lot $\mathrm{HH}$, pour un même niveau de rationnement (865 contre 7 I $3 \mathrm{~g} / \mathrm{jour}$ ). L'élevage des femelles en claustration totale, dans un bâtiment sans fenêtre, peut aussi être à l'origine du retard d'apparition de la puberté, comme 1'avait indiqué SALMON-LEGAGNEUR (I970).

Dans ces conditions expérimentales, une sous-alimentation au moment de la croissance accroit 1'âge à la puberté, ce qui est en accord avec HAINEs et al. (I959), mais l'écart de deux mois environ entre l'âge au premier œestrus des truies des deux lots est trois fois plus élevé que celui rapporté par ces auteurs. Par contre, si l'abaissement du niveau alimentaire est moins sévère (20 à $30 \mathrm{p}$. Ioo du niveau libéral), i1 ne subsiste plus de retard à la puberté (LODGE et McPherson, I96I). Dans tous les cas, et en particulier dans la présente expérience, les truies nourries d'une manière libérale en croissance ont, à la puberté, un poids plus élevé.

Parallèlement à cette augmentation du poids à la puberté chez les truies recevant un niveau alimentaire élevé en croissance, il a été établi un accroissement significatif de I, 8 ovules pondus, ce qui est conforme aux résultats de SELF et al. (I956) ou de Haines et al. (I959). D'ailleurs, une liaison linéaire relie, étroitement, le taux d'ovulation et le poids à la puberté et ceci a déjà été montré par RATHNASABAPATHy et al. (I956). Si le coefficient de régression de cette relation calculé sur la population totale est de 0,059, il est différent, mais non significativement, entre les deux groupes de truies, étant plus élevé $(0,076)$ dans le groupe restreint que dans l'autre groupe $(0,0,2)$. Cette remarque pourrait indiquer l'existence d'un plateau, au-dessus duquel le taux d'ovulation n'augmente plus avec le poids maternel au moment de l'œstrus.

L'accroissement du taux d'ovulation se répercute, dans le cas présent, sur le nombre d'embryons viables à 30 jours. Mais la différence de 3,5 embryons entre les lots haut et bas indique qu'en outre une mortalité embryonnaire plus élevée se produit chez les truies restreintes pendant la période prépubérale. Ces résultats sont à rapprocher des observations de PIKE et BOAZ (I972), montrant l'importance de l'état nutritionnel de la truie au moment de la saillie sur la mortalité embryonnaire ultérieure; de tels effets sur le nombre d'embryons viables ne sont pas retrouvés par HaInes et al. (I959), LodGe et McPherson (I96I). Il faut toutefois signaler que la mortalité embryonnaire est anormalement faible chez les femelles recevant une alimentation libérale avant la saillie $(8,8 \mathrm{p}$. Ioo).

Finalement, en retardant l'âge à la puberté et en abaissant le nombre d'embryons viables à 30 jours de gestation, une restriction alimentaire importante pendant la croissance a un effet néfaste sur les performances de reproduction chez la Truie. 


\section{2. - Influence du niveau alimentaire de gestation}

Si le niveau alimentaire prépubéral affecte le nombre d'embryons à un mois de gestation, il n'en est pas de même du niveau alimentaire appliqué après la saillie. En particulier, la mortalité embryonnaire n'est pas diminuée quand on augmente la consommation des animaux en gestation, ce qui est en accord avec les résultats expérimentaux de McGilimivay et al. (I963), de Schulitz et al. (I966) ou de Pike et BOAZ (I972), mais reste en contradiction avec ceux de Goope et al. (I965).

Le poids moyen corrigé des embryons à 30 jours de gestation n'est pas modifié par le niveau de consommation de la mère. Ce résultat est logique quand on connait la modicité des dépôts qui sont affectés aux fœetus pendant le premier mois qui suit 1'accouplement chez la Truie (Mous'TGaARD, I962). D'ailleurs, si SALMON-LEGAGNEur et al. (I966), trouvent une corrélation hautement significative de $-0,48$ entre la taille de la portée et le poids moyen des porcelets à la naissance, BAKER et al. (I958) ou LEGAUL,T et LEUILLET (I973) n'obtiennent pas de relation semblable 25 ou 30 jours après l'accouplement. Il n'y aurait donc pas de concurrence nutritionnelle entre les embryons au début de la gestation.

Par contre, par une diminution du poids moyen des corps jaunes et du poids total des ovaires, une restriction alimentaire pendant la gestation pourrait perturber la sécrétion d'hormones liée à ce stade physiologique (la progestérone, en particulier) dans la mesure où la quantité secrétée est proportionnelle au poids du corps jaune (RoMBauts et al., I97I).

Bien qu'aucune différence de mortalité embryonnaire n'apparaisse à 30 jours de gestation, ceci pourrait peut être remettre en cause, ultérieurement, la survie fœtale ou le déroulement normal de la gravidité.

De même, un niveau bas de consommation au cours de la gestation modifie l'environnement intra-utérin de la portée, en diminuant la quantité de tissus placentaires disponibles par embryon et le poids total des liquides allantoïdiens.

Dans tous les cas, l'effet de la sous-alimentation est plus nuancé si le niveau alimentaire avant la saillie est libéral.

En conclusion, il apparait qu'au stade de gestation étudié (3o jours) le nombre et le poids des embryons ne sont pas affectés par le niveau de consommation maternel. En revanche, 1'abaissement du niveau alimentaire durant les 30 premiers jours de gestation a pour effets de diminuer la quantité d'annexes disponibles par embryon et le poids des ovaires, comme le poids moyen des corps jaunes.

$$
\text { Rę̧u pour publication en juin } 1973 .
$$

\section{SUMMARY}

EFFECT OF DIFFERENT FEEDING LEVELS DURING GROWTH

AND THE FIRST MONTH OF PREGNANCY ON REPRODUCTIVE PERFORMANCES IN THE GILT

The experiment was made on 2 groups of 12 Large White gilts (from a live weight of $55 \mathrm{~kg}$ reached at a mean age of $\mathrm{I} 55$ days) full hand-fed (maximum intake; $3.28 \mathrm{~kg} / \mathrm{day}$ ) or subjected to restricted feeding (about $45 \mathrm{p}$. roo of the food intake). The gilts were mated at puberty and each main groups was subdivised into 2 groups of 6 animals each. The feeding level was maintai- 
ned in the first group, whereas the second group was subjected to the other feeding level during the first month of pregnancy. At the end of this period, the animals were slaughtered, and the reproductive tract removed. mating.

No significant interaction was noted between the effects of feeding levels before and after

The daily weight gain of the sows during growth or pregnancy was parallel to their food intake.

Undernutrition during growth delayed by 2 months the age at puberty (363 versus 296 days) and lowered their weight at this stage (I 20,6 versus $147.4 \mathrm{~kg}$ ). The poor results concerning age at puberty of the two groups might be explained by their bad performances before allotment and also by their housing in total confinment. In addition, the ovulation rate regressed significantly in the restricted gilts (12.0 versus 13.8 ). This may be due to the lighter weight of these animals : a linear regression was found between the ovulation rate $(Y)$ and the weight $(X, \mathrm{~kg})$ at puberty of the sows $(\mathrm{Y}=0.059 \mathrm{X}+4.99 ; \gamma=0.53)$, and after statistical adjustment to the same weight of $135 \mathrm{~kg}$, no more difference was noted (I 2.7 versus I3. 1 ). The difference in number of ova shed had a repercussion on the number of viable embryos at 30 days of pregnancy (9.0 versus 12.5$)$. Embryonnic mortality appeared also to be influenced by the feed intake before puberty; it was higher in restricted gilts (26.0 versus 8.8 p. Ioo)

The feeding level during pregnancy ( 1.8 or $3.5 \mathrm{~kg}$ per day) did not affect the number and weight of the embryos at 30 days. On the contrary, lowering of the food intake during this period decreased the amount of material available per embryo (placental membranes, allantoid fluids), and also the mean weight of corpora lutea from 548 to $416 \mathrm{mg}$. The significance of this result on maintenance of pregnancy is discussed.

\section{RÉFÉRENCES BIBLIOGRAPHIQUES}

Baker L. N., Chapman A. B., Grummer R. H., Casida L. E., i958. Some factors affecting litter size and fetal weight in purebred and reciprocal-cross matings of Chester White and Poland China swine. J. Anim. Sci., 17, 612-621.

Goode L., Warnick A. C., Wallace H. D., 1965. Effect of dietary energy levels upon reproduction and the relation of endometrial phosphatase activity to embryo survival in gilts. J.Anim. Sci., 24, 959-963.

Gossett J. W., Sorensen A. M., I959. The effects of two levels of energy and seasons on reproductive phenomena of gilts. J. Anim. Sci., 18, 40-47.

Haines C. E., Warnick A. C., Wallace H. D., I959. The effect of two levels of energy intake on reproductive phenomena in Duroc Jersey gilts. J. Anim. Sci., 18, 347-354.

Legault C., I973. Déterminisme génétique de la précocité sexuelle, du taux d'ovulation, et du nombre d'embryons chez la truie primipare : héritabilité, effet d'hétérosis. Journées Rech. Porcine en France, Paris, I $47-154$, ed. by I. T. P.

Legault C., Leuillet M., I973. Étude de quelques facteurs de variation du poids de l'embryon et du placenta chez la Truie primipare au trentième jour de la gestation. Ann. Biol. anim. Bioch. Biophys., 13, 25-36.

Lodge G. A., McPherson R. M., I96r. Level of feeding during early life and the subsequent reproductive performance of sows. Anim. Prod., 3, 19-28.

McGillivray J. J., Nalbandov A. V., Jensen A. H., Norton H. W., Harmon B. G., Becker D. E. 1963. Effect of changing energy intake on reproductive performance in gilts. J. Anim. Sci., 22, I 127 (Abstr.).

Moustgaard J., I962. Fotal nutrition in the pig. In Morgan, Lewis, Nutrition of pigs and poultry, Butterworths, London, I89-205.

PIKE I. H., BoAZ T. G., 1972. The effect of condition at service and plane of nutrition in early pregnancy in the sow. I. Uterine and extra-uterine changes. Anim. Prod., 15, 147-155.

Rathnasabapathy V., Lasley J. F., Mayer D. T., I956. Genetic and environmental factors affecting litter size in swine. Univ. Missouri A gric. Exp. Sta. Res. Bull. no 615.

Rombauts P., Fevre J., Terqui M., I97I. (Estrogènes et progestogènes au cours du cycle de reproduction de la Truie. Journées Rech. Porcine en France, Paris, I73-178, éd. by I. T. P.

Salmon-Legagneur E., Legault C., Aumaitre A., 1966. Relations entre les variations pondérales de la Truie en reproduction et les performances d'élevage. $A n n$. Zootech., 15, 215-229.

Salmon-Legagneur E., 1970. Étude de quelques facteurs de variation de l'âge et du poids des truies Large White au premier westrus. Journées Rech. Porcine en France, Paris, 4I-46, éd. by I. T. P.

Schultz J. R., Speer V. C., Hays V. W., Melampy R. M., I966. Influence of feed intake and progesterone on reproductive performance in swine. J. Anim. Sci., 25, 157-160. 
Self H. L., Grummer R. H., Casica L. E., I955. The effects of various sequences of full and limited feeding on the reproductive phenomena in Chester White and Poland China gilts. J. Anim. Sci., 14, 573-592.

Sørensen A. M. Jr., Thomas W. B., Gossett J. W., ig6r. A further study of the influence of level of energy intake and season on reproductive performances of gilts. J. Anim. Sci., 20, 347-349. 